\title{
Micro- and nanotechnology for isolation and detection of circulating tumor cells
}

\author{
Nam-Trung Nguyen ${ }^{\mathrm{a}^{*}}$ \\ ${ }^{a}$ Queensland Micro- and Nanotechnology Centre, Griffith University, Brisbane, Qld 4111, Australia
}

\begin{abstract}
Circulating tumor cells (CTC) are rare cells, which are shed from a heterogeneous primary tumor into circulation. Although there is only one CTC for every one billion peripheral blood cells, these cells spread the cancer through metastasis to secondary sites. Isolating and analyzing CTCs are challenging tasks that potentially can be solved with advances in micro- and nanotechnologies. Analyzing the isolated CTCs help to understand their role in the spreading of cancer and ultimately lead to successful treatment of the disease.
\end{abstract}

Keywords: circulating tumor cells, microfluidics, biomarkers, deformability, cancer treatment, personalized medicine

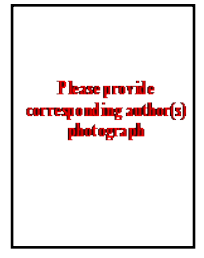

In the past decade, various technologies have been developed for isolating and analyzing circulating tumor cells (CTC) [1, 2]. Microfluidic technology is a powerful tool for this task [3]. In general, the isolation of CTCs can be categorized according to their immunochemical and physical signatures. Immunochemical techniques recognize and CTCs based on markers on the cell surfaces such as the epithelial cell adhesion molecule (EpCAM). Physical techniques are based on the various physical signatures of the CTCs such as size, density, deformability, charges and magnetic properties.

As mentioned above, the markers on the cell surfaces form the immunochemical signature for the isolation of CTCs. A microchannel surface coated with antibodies is able to capture the CTC through antigen-antibody bonds. Immunochemical techniques are further categorized as positive and negative isolation. Positive isolation techniques capture and retain the CTCs in the device, while negative techniques capture all other cell types and let CTCs to be collected at the exit.

Physical techniques separate CTCs according to size, density, deformability, dieletrophoretic and magnetic properties. CTCs are larger than blood cells and can be separated from them using inertial microfluidics with high throughput [4]. Inertial microfluidics separate CTCs in the regime of relatively high flow velocities. In this regime, inertial effects such as inertial migration and secondary flow

\footnotetext{
* Correspondence to:

Nam-Trung Nguyen, Queensland Micro- and Nanotechnology Centre, Griffith University, Brisbane, QLD 4111, Australia. Email: namtrung.nguyen@griffith.edu.au
}

become more significant. The effect of inertial migration occurs where particles in a straight microchannel migrate and retain equilibrium positions in the channel cross section.
This effect results from the balance between the shear gradient lift force generated from the velocity distribution and the wall lift force [4]. The effect of secondary flow occurs if the flow changes direction in cases such as flown in a curved channel or a straight channel with obstacles. Sizedependent separation based on the equilibrium of inertial lift force and secondary flow drag allows for the isolation of CTCs from other blood cells. Current challenges for the isolation of CTCs using inertial microfluidics are the broad size distributions of CTCs and the dynamic nature of these cells. Their size may change during the circulation.

Deformability is a physical signature that has been used for filtering and detecting diseased cells such as malaria infected red blood cells. Bagnall et al. [5] recently reported the trapping and detection of CTCs using a technology called Suspended Microchannel Resonator (SMR). CTCs of the same size as blood cells that cannot be detected with sizebased techniques can be probed using deformability. A SMR is an U-shape microchannel embedded in a cantilever. The change in the buoyant mass of the microchannel will lead to a shift of the resonant frequency of the cantilever. Placing a constriction to the microchannel allows for the measurement of deformability of cells passing through the system. More deformable cells will pass through the constriction faster leading to a shorter passage time, which can be measured from the time history of the resonant frequency. Thus, the SMR system can determine both size and deformability in a single experiment through resonant frequency and passage time, respectively.

Different cell types have their distinct membrane capacitance and can be separated and isolated using dielectrophorsis, where the field strength and the alternating current (AC) frequency of the applied field can be used to tune the selective isolation. The difference in magnetic susceptibility of cells can be utilized for isolation using either 
magnetophoresis - the migration of magnetic particles in a diamagnetic medium or diamagnetophoresis - the motion of diamagnetic particles in a magnetic medium [6]. If the intrinsic magnetic signature of the cells is not strong enough, immunospecific bonding to magnetic nano- or micro particles can make the target cells magnetic and increases the magnetophoretic force for effective isolation. Issadore et al. [7] used magnetic sensors integrated in a microchannels to detect and count CTCs labeled with magnetic nanoparticles. The magnetic sensor detects the magnetic moment of the nanoparticles. Similar to the device proposed by Bagnall et al. [5], the combination of microfluidics with an integrated sensor allows the measurement of CTCs in blood at singlecell resolution.

The availability of micro-nanotechnology for isolation and detection of CTCs allows these cells to potentially work as both predictive and prognostic biomarkers to inform physicians on tumor progression and to adjust therapeutic treatment. A prognostic biomarker provides a risk assessment for the cancer patient such as disease progression, recurrence, and patient's survival. A predictive biomarker provides a feedback on the disease response from a therapeutic treatment. To date, no CTC-based technique has a predictive capability. The isolated CTCs may also be useful for drug screening. Isolated CTCs can serve as a pharmacodynamics marker for a specific molecular target. Furthermore, isolated CTCs could act as a biomarker to monitor the effects of cancer drugs. The number of detected CTCs could predict the overall survival of a cancer patient.
In summary, micro- and nanotechnology open new avenues for the isolation and detection of CTCs. These technologies would allow for the establishment of CTC assays that inform the current state of the disease and assess its response to treatment therapies. The analysis of isolated CTCs at an early stage of a treatment process could predict the potential benefit of the treatment therapy. This early assessment could help to stop an ineffective treatment, minimizing patient's suffering and timely providing a treatment alternative. CTCbased technology potentially allows for continuing assessment and personalizing the treatment for patients with advanced cancer.

\section{REFERENCES}

[1] Lianidou ES, Markou A, Circulating tumor cells in breast cancer: Detection systems, molecular characterization, and future challenges. Clin. Chem. 2011; 57:1242-1255.

[2] Lang JM, Casvant BP, Beebe DJ, Circulating tumor cells: getting more from less. Sci Trans. Med. 2012; 4: 141ps13.

[3] Chen YC, Li P, Huang PH, Xie YL, Mai JD, Nguyen NT, Huang TJ, Rare cell isolation and analysis in microfluidics, Lab Chip 2014; 14:626645.

[4] Zhang J, Yan S, Yuan D, Alici G, Nguyen NT, Warkiani ME, Li WH, Fundamentals and applications of inertial microfluidics: a review, Lab on a Chip, 2016; DOI: 10.1039/C5LC01159K

[5] Bagnall JS, Byun S, Begum S, Miyamoto DT, Hecht VC, Maheswaran S, Stott SL, Toner M, Hynes RO, Manalis SR, Deformability of tumor cells versus blood cells, Scientific Reports, 2015; 5:18542

[6] Hejazian M, Li WH, Nguyen NT, Lab on a chip for continuous-flow magnetic cell separation, Lab on a Chip 2015; 15:959-970.

[7] Issadore D, Chung J, Shao H, Liong M, Ghazani AA, Castro CM, Weissleder $\mathrm{R}$, Lee $\mathrm{H}$, Ultrasensitive clinical enumeration of rare cells ex vivo using a micro-hall detector, Sci Transl Med 2012; 4:141ra92 